\title{
Regulation of Corticosteroidogenic Genes by MicroRNAs
}

\author{
Stacy Robertson, ${ }^{1}$ Louise A. Diver, ${ }^{1}$ Samantha Alvarez-Madrazo, ${ }^{1}$ Craig Livie, ${ }^{1}$ Ayesha Ejaz, ${ }^{1}$ \\ Robert Fraser, ${ }^{1}$ John M. Connell, ${ }^{2}$ Scott M. MacKenzie, ${ }^{1}$ and Eleanor Davies ${ }^{1}$
}

${ }^{1}$ Institute of Cardiovascular and Medical Science, University of Glasgow, Glasgow, UK

${ }^{2}$ Ninewells Hospital and Medical School, University of Dundee, Dundee, UK

Correspondence should be addressed to Eleanor Davies; eleanor.davies@glasgow.ac.uk

Received 29 March 2017; Revised 30 May 2017; Accepted 18 June 2017; Published 9 August 2017

Academic Editor: Carmela Maniero

\begin{abstract}
Copyright ( 92017 Stacy Robertson et al. This is an open access article distributed under the Creative Commons Attribution License, which permits unrestricted use, distribution, and reproduction in any medium, provided the original work is properly cited.
\end{abstract}

\begin{abstract}
The loss of normal regulation of corticosteroid secretion is important in the development of cardiovascular disease. We previously showed that microRNAs regulate the terminal stages of corticosteroid biosynthesis. Here, we assess microRNA regulation across the whole corticosteroid pathway. Knockdown of microRNA using Dicer1 siRNA in H295R adrenocortical cells increased levels of CYP11A1, CYP21A1, and CYP17A1 mRNA and the secretion of cortisol, corticosterone, 11-deoxycorticosterone, 18-hydroxycorticosterone, and aldosterone. Bioinformatic analysis of genes involved in corticosteroid biosynthesis or metabolism identified many putative microRNA-binding sites, and some were selected for further study. Manipulation of individual microRNA levels demonstrated a direct effect of miR-125a-5p and miR-125b-5p on CYP11B2 and of miR-320a-3p levels on CYP11A1 and CYP17A1 mRNA. Finally, comparison of microRNA expression profiles from human aldosterone-producing adenoma and normal adrenal tissue showed levels of various microRNAs, including miR-125a-5p to be significantly different. This study demonstrates that corticosteroidogenesis is regulated at multiple points by several microRNAs and that certain of these microRNAs are differentially expressed in tumorous adrenal tissue, which may contribute to dysregulation of corticosteroid secretion. These findings provide new insights into the regulation of corticosteroid production and have implications for understanding the pathology of disease states where abnormal hormone secretion is a feature.
\end{abstract}

\section{Introduction}

Cardiovascular disease is a major cause of mortality and morbidity and therefore a key public health issue. The role of the corticosteroids, aldosterone and cortisol, in the modulation of blood pressure is well known. Their gross excess results in hypertension, and this can be ameliorated by pharmacological or surgical targeting of the production and action of these steroid hormones [1,2]. Even in essential hypertension, blockade of these hormones lowers blood pressure [3]. However efforts to develop specific aldosterone synthase inhibitors is limited, mostly due to its similarity to $11 \beta$-hydroxylase. An alternative approach to effectively reduce aldosterone production may be to target regulators of CYP11B2 expression. Currently, our understanding of the various mechanisms that control corticosteroid biosynthesis in normal and pathological states is incomplete. This must be improved if we are to develop more effective hypertension treatments.

Cortisol and aldosterone are the final products of a series of enzyme-controlled reactions that occur in the adrenal cortex. Following transport of cholesterol to the mitochondrion, several cytochrome mixed function oxidases and hydroxysteroid dehydrogenases, each encoded by specific genes, control the integrated series of reactions (Figure 1) culminating in the production of cortisol by $11 \beta$-hydroxylase (CYP11B1) and aldosterone by aldosterone synthase (CYP11B2) [4]. Cortisol can also be reversibly converted to cortisone by $11 \beta$-hydroxysteroid dehydrogenases types 1 and 2 (HSD11B1, HSD11B2) [5, 6]. Some intermediate 


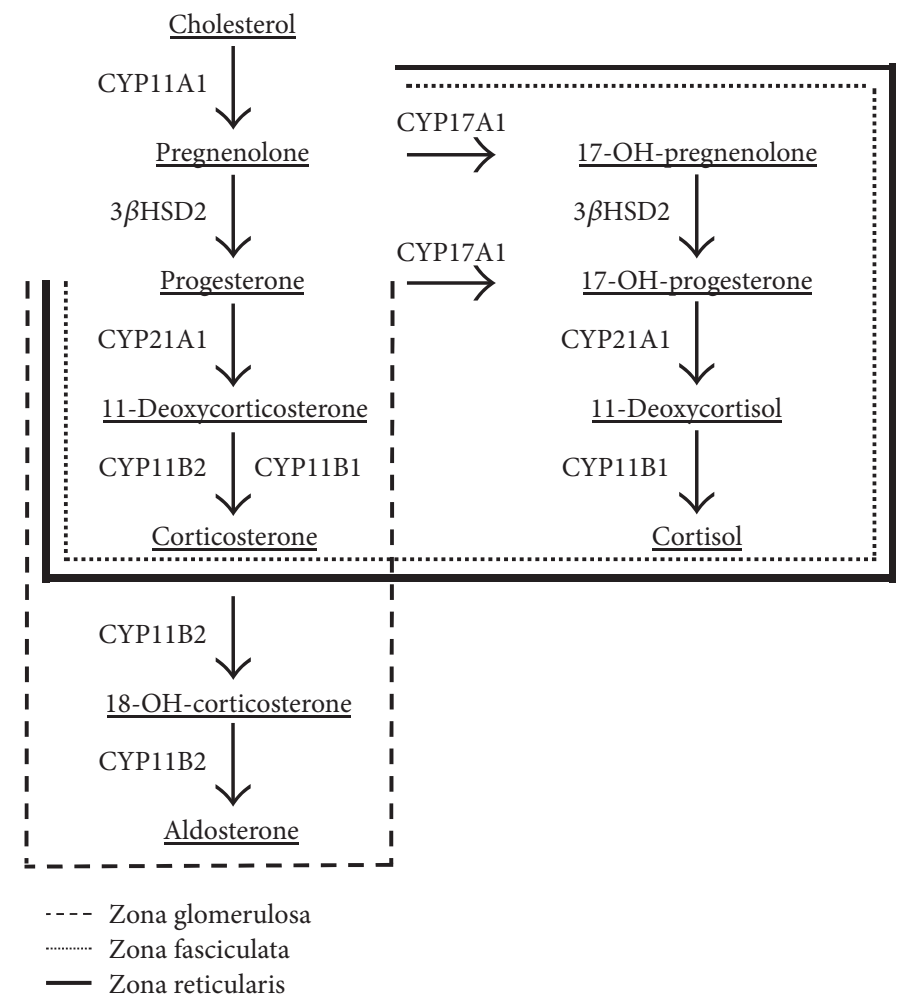

Figure 1: Pathway of corticosteroid synthesis in the adrenal cortex. Dashed lines represent the zone of the adrenal cortex in which each reaction occurs.

products in these pathways can themselves have deleterious cardiovascular effects, such as the mineralocorticoid, 11-deoxycorticosterone [7].

Expression of corticosteroidogenic enzymes is tightly controlled. This is regulated predominantly at the transcriptional level, but studies by ourselves and others indicate that posttranscriptional modification by microRNA (miRNA) also plays a significant role [8-11]. miRNAs are endogenous small noncoding RNA molecules cleaved from intronically or intergenically located primary miRNA (pri-miR) sequences. Their transcription, in the form of a pri-miR, may be controlled via host gene promoter activity or through a separate miRNA promoter region [12]. They mature via a hairpin structure called a pre-miR, to the final miRNA, this last step being catalysed by the Dicer enzyme [13]. Mature miRNAs are incorporated into the RNA-induced silencing complex (RISC) and use their specific sequence to bind imperfectly to the $3^{\prime}$ untranslated region (UTR) of target mRNA. The RISC contains several endonucleases which can repress target mRNA levels through either translational repression or mRNA degradation. Hence, miRNAs can fine-tune levels of mRNA and contribute to cellular homeostasis [14]. Each step in the corticosteroidogenic pathway represents a potential target for miRNA regulation. Furthermore, a single miRNA is capable of regulating several constituents of a single pathway [15] so that the sum of many small individual effects could result in pronounced changes overall.

Abnormal expression of specific miRNAs can be pathogenic $[14,16]$; miRNA levels are altered in adrenal carcinoma and adenoma tissue when compared with normal adrenal tissue [8, 17-23]. However, only a limited number of studies have examined regulation of corticosteroidogenic genes by miRNAs. Previously, we showed that knockdown of Dicer 1 disrupted CYP11B1 and CYP11B2 mRNA levels in adrenocortical cells in vitro, pointing to a regulatory role for miRNAs [8]. We identified miR-24 as one such miRNA due-at least in part-to its regulatory effects on CYP11B1 and CYP11B2 expression [8]. However, the effect of this individual miRNA on total corticosteroid biosynthesis is small and numerous other miRNAs are likely to affect this pathway and probably not only at the terminal points represented by these two genes $[8,10,11]$. The combined regulatory impact of miRNAs simultaneously across all the components of the corticosteroid pathway remains to be assessed.

In this study, we expand the scope of our investigations to encompass all of the genes directly contributing to corticosteroid production, including CYP11A1 and CYP17A1 (Figure 1). We demonstrate that Dicer1 knockdown by siRNA affects the corticosteroidogenic pathway in vitro at numerous points, earlier than the terminal reactions we had previously reported, and that these effects are apparent at both the mRNA and the steroid levels. We then analyse the effects of individual miRNAs on specific transcripts, guided by bioinformatic analysis of gene sequence. Finally, we demonstrate that certain of these corticosteroid-regulating miRNAs are differentially expressed in aldosterone-producing adenoma tissue (APA). We propose that these miRNAs may have a significant pathogenic role and potential diagnostic value. 
TABLE 1: Sequences of primers used in qRT-PCR assays.

\begin{tabular}{lcc}
\hline Gene & Forward primer & Reverse primer \\
\hline$\beta$-Actin & CCAACCGCGAGAAGATGA & CCAGAGGCGTACAGGGATAG \\
GAPDH & GCTCTCTGCTCCTCCTGTTC & ACGACCAAATCCGTTGACTC \\
StAR & TACGTGGCTACTCAGCATCG & ACCTGGTTGATGATGCTCTTG \\
CYP11A1 & AGGAGGGGTGGACACGAC & TTGCGTGCCATCTCATACA \\
CYP21A1 & GCTCCAGGAAGCGATCAG \\
CYP17A1 & GAGGGCACAGTCATCATTCC & TTGTCCACAGCAAACTCACC \\
HSD3B2 & CTATGCTCATCCCCCACAG & CCTCAAGTACAGTCAGCTTGG \\
CYP11B1 & GGGCCTTCAGACCAGAATTG & GGCAGCATCACACACACC \\
CYP11B2 & ACTAGGGCCCATTTTCAGGT & CACACACCATGCGTGGTCC \\
HSD11B2 & GCACCTGCACCTGGAGATG & CACTGACCCACGTTTCTCAC \\
\hline
\end{tabular}

\section{Materials and Methods}

2.1. Cell Culture and Transfection. The H295R human adrenocortical cell line (a gift from Professor William Rainey, Medical College of Georgia, USA) [24] and HeLa cells (European Collection of Animal Cell Cultures, Wiltshire, UK) were maintained as previously described [8] and used between passage numbers 15 and 25. Cells were transfected using siPORT NeoFX Transfection Agent (Applied Biosystems, Warrington, UK) according to the manufacturer's instructions; H295R cells were seeded to a final density of $4.8 \times 10^{5}$ cells/well in 6 -well plates and HeLa cells to $8 \times 10^{4}$ cells/well in 24-well plates. Pre-miR ${ }^{\mathrm{TM}}$ or Anti-miR ${ }^{\mathrm{TM}}$ molecules (miR-125a-5p: product code 12561; miR-125b-5p miR-134-3p 10341; miR-495-3p: 11526; and miR-320a-3p: 11621, Applied Biosystems) were transfected to a final concentration of $50 \mathrm{nM}$ and prevalidated siRNA molecules (Dicer 1A: product code s23755; Dicer 1B: s23756, Applied Biosystems) to a final concentration of $30 \mathrm{nM}$. Reporter constructs were cotransfected with pEZX construct (500 ng) and either a Pre-miR or an Anti-miR.

2.2. RNA Isolation and Real-Time Quantitative Reverse Transcriptase PCR ( $q R T-P C R)$. Total RNA was isolated from H295R cells using the miRNeasy mini kit (QIAGEN, Crawley, UK) according to the manufacturer's instructions. First-strand cDNA was synthesised in a $20 \mu \mathrm{L}$ volume using the miScript RT kit (QIAGEN) and $200 \mathrm{ng}$ of total RNA. The resulting cDNA was diluted to a final volume of $100 \mu \mathrm{L}$ of which $2 \mu \mathrm{L}$ was amplified by qRT-PCR on the ABI PRISM 7900HT apparatus (Applied Biosystems). Reactions used the miScript SYBR Green PCR kit (QIAGEN) to measure mature miRNA-derived cDNA sequences and the Universal ProbeLibrary System (Roche Applied Science, Indianapolis, USA) and ABsolute ${ }^{\mathrm{TM}}$ QPCR ROX Mix (Abgene, Epsom, $\mathrm{UK})$ to measure mRNA cDNA using specific primer sequences (Table 1).

2.3. Steroid Measurement. Steroids were extracted from cell media using ChemElute cartridges (Varian) and eluted from the cartridge with dichloromethane. The eluates were evaporated to dryness and reconstituted in $10 \%$ acetonitrile. Identification and quantification of steroid products was achieved by tandem mass spectrometry using a Varian $1200 \mathrm{~L}$ mass spectrometer with a triple quadrupole detector [25].

2.4. Identification of the Genomic Location of miRNAs and Prediction of miRNA Targets. The genomic coordinates, strand location, and mRNA transcript length of the human genes encoding corticosteroidogenic enzymes were identified using the Ensembl Genome Browser (release 79, March 2015). These were cross-referenced with known miRNA precursor sequences mapped and stored in the miRBase database (release 21, June 2014). Putative miRNA target sites in the 3'UTRs of corticosteroidogenic genes were identified by four commonly used prediction algorithms (MicroCosm Targets (v.5), microRNA.org (Nov 2010), miRviewer (June 2005), and TargetScan (v. 6.2)).

2.5. Reporter Construct Studies. A luciferase reporter construct was purchased from LabOmics (Nivelles, Belgium) to determine miRNA binding to the 3'UTR. The construct (pEZX-B2) contained a pEZX reporter backbone, which comprises a renilla reporter gene coupled to a SV40 viral promoter, a firefly experimental gene coupled to a CMV promoter and the full-length 3'UTR sequence of the CYP11B2 gene. This was cotransfected with Pre-miR or Anti-miR into HeLa cells; a "no-3'UTR insert" vector (pEZX-C) was used as a control (LabOmics). Firefly and renilla luciferase activity was measured 48 hours posttransfection using the Dual Luciferase Reporter Assay system (Promega, Madison, USA) and a Lumat LB 9507 tube luminometer (Berthold Technologies, Harpenden, UK).

2.6. Human Adrenal Gland miRNA Microarray Analysis. Four frozen nondiseased predominantly cortical adrenal tissue samples were obtained from white adult patients undergoing nephrectomy, with full local ethical approval from the University of Birmingham, UK. Four samples of formalin-fixed paraffin-embedded (FFPE) APA tissue were obtained from the University of Glasgow Biobank. Use of tissue in this study was conducted in accordance with the requirements of the Human Tissue Act and with appropriate permission from the local ethical review board. Participants gave informed consent. Total RNA from $40 \mathrm{mg}$ frozen tissue 
samples or from four $20 \mu \mathrm{m}$ FFPE tissue sections was prepared using the miRNeasy mini kit (QIAGEN) or the RecoverAll $^{\mathrm{TM}}$ Total Nucleic Acid Isolation kit (Applied Biosystems) according to the manufacturer's instructions, respectively. miRNA microarray analyses for 723 miRNAs (miRBase v 10.1) were performed by LC Sciences (Houston, Texas, USA), using $5 \mu \mathrm{g}$ of the total RNA, as previously described [8]; selected data from this study was previously published [8].

2.7. Data Analysis. qRT-PCR results were analysed using the relative quantification method of comparative $C_{t}\left(\Delta \Delta C_{t}\right)$ [26]. In vitro results were analysed using either an unpaired Student's $t$-test or one-sample $t$-test as stated. Statistical analysis was performed using Graph Pad Prism 6.0 software and significance reached when $p<0.05$. All results are expressed as mean \pm standard error of the mean (SEM). Unless stated otherwise, in vitro experiments were performed in at least three technical replicates, on three biologically independent occasions $(n=3)$.

\section{Results}

3.1. siRNA Knockdown of Dicer1 Increases Levels of Selected Steroidogenic mRNAs and Steroid Secretion in H295R Cells. Significant knockdown of Dicer1 mRNA by both Dicer1A and Dicer $1 B$ siRNA transfection into the H295R adrenocortical cell line was demonstrated $(0.56 \pm 0.07$-fold and $0.50 \pm 0.06$-fold, resp.; $p<0.001$ for each versus scrambled control siRNA) [8]. Total RNA was isolated 48 hours posttransfection from cells transfected with scrambled control siRNA or Dicer1A siRNA; levels of mRNAs were measured by real-time qRT-PCR (Figure 2). Significant reduction in Dicer $1 \mathrm{mRNA}$ level did not significantly affect the abundance of $S t A R, 3 \beta H S D I I$, or HSD11B2 mRNAs, but those of the three cytochrome P450-encoding mRNA (CYP11A1, CYP21A1, and CYP17A1) were all significantly increased relative to cells transfected with a scrambled control siRNA $(1.41 \pm 0.09$-fold $p<0.05 ; 2.40 \pm 0.34$-fold, $p<0.01$; and $1.73 \pm 0.22$-fold, $p<0.01$, resp.). Steroid secretion from H295R cells was measured by LCMS MS:MS 48 hours after transfection with a scrambled control or Dicer1B siRNA (Figure 3). Knockdown of Dicer1 significantly increased secreted levels of cortisol (1.33 \pm 0.11 fold; $p=0.01)$, corticosterone (1.32 \pm 0.13 -fold; $p=0.03)$, 11 -deoxycorticosterone $(1.53 \pm 0.09$-fold; $p<0.001), \quad 18$ hydroxycorticosterone $(1.29 \pm 0.10$-fold; $p=0.04)$, and aldosterone $(1.47 \pm 0.11$-fold; $p<0.01)$ relative to scrambled siRNA transfected cells. Levels of 11-deoxycortisol and cortisone rose but did not achieve statistical significance.

3.2. Corticosteroidogenic Genes Contain Putative $3^{\prime} U T R$ miRNA Target Sites. Putative miRNA binding sites were identified in the 3 'UTR in each of the seven human corticosteroidogenic genes (CYP11B1, CYP11B2, CYP11A1, CYP17A1, CYP21A2, HSD3B2, and StAR) and in the two metabolising genes (HSD11B1, HSD11B2) analysed (Table 2). CYP11B1 has the longest 3'UTR (2022 base pairs) and contains the highest number of putative miRNA binding sites
(390 sites). Conversely, CYP17A1 has the shortest 3'UTR and the lowest number of predicted miRNA binding sites (59 sites).

3.3. The miR-125 Family Regulates CYP11B2 mRNA Expression. In order to identify individual miRNAs contributing to the net miRNA effect, as observed under Dicer1 knockdown, four miRNAs (miR-125a-5p, miR-125b-5p, miR-134-3p, and miR-495-3p) expressed in human adrenal tissue and with putative binding sites in the $3^{\prime}$ UTR of CYP11B2 were selected for further study. Luciferase reporter constructs containing the 3'UTR of CYP11B2 (pEZX-B2) were transfected into HeLa cells alongside Pre-miR or AntimiR molecules specific to each of these miRNAs. Levels of luciferase activity were then measured (Figures 4(a), 4(b), $4(c)$, and 4(d)). Manipulation of miR-134-3p or miR-495$3 p$ levels in this manner did not significantly affect the luciferase activity of the pEZX-B2 reporter construct. However, the presence of active binding sites predicted for miR-125a-5p and miR-125b-5p was confirmed by changes in luciferase activity. Increasing the levels of miR-125a-5p in Pre-miR-transfected cells reduced luciferase activity to $63.78 \pm 8.70 \%(p=0.011)$ while reduction of miR125a-5p levels in Anti-miR-transfected cells significantly increased luciferase activity to $173.72 \pm 22.54 \%(p=0.040)$. Similarly, miR-125b-5p Pre-miR significantly decreased luciferase activity $(75.90 \pm 1.28 \% ; p=0.033)$, while its Anti$\mathrm{miR}$ resulted in a significant increase (156.39 $\pm 14.26 \%$; $p=0.017)$. These responses are consistent with canonical miRNA action.

To determine the direct effect of these miRNAs on CYP11B2 mRNA expression, Pre-miR or Anti-miR molecules were then transfected into the H295R adrenocortical cell line. Here, miR-125a-5p Pre-miR decreased CYP11B2 mRNA abundance $(0.69 \pm 0.002$-fold; $p<0.0001)$, while its Anti-miR significantly increased it $(1.62 \pm 0.014$-fold $p=0.011$; Figure 4(e)). miR-125b-5p Pre-miR transfection reduced CYP11B2 mRNA to $0.75 \pm 0.098$-fold of control levels, but this did not attain statistical significance $(p=0.069)$. However, miR-125b-5p levels with Anti-miR did significantly increase CYP11B2 mRNA to $1.36 \pm 10.04$ fold $(p=0.041)$ of control levels (Figure 4(f)).

3.4. miR-320a Is a Common Regulator of CYP17A1 and CYP11A1. Bioinformatic analysis predicted a miRNA binding site for miR-320a-3p in the 3'UTR of two corticosteroidogenic genes: CYP11A1 and CYP17A1. This was tested by transfecting H295R cells with miR-320a-3p Pre-miR and Anti-miR molecules and then measuring mRNA 48 hours posttransfection by qRT-PCR (Figure 5). Raising miR$320 \mathrm{a}-3 \mathrm{p}$ led to significantly decreased CYP11A1 mRNA $(0.81 \pm 0.02$-fold; $p<0.05)$ but did not significantly affect CYP17A1 (1.02 \pm 0.12 -fold). Reduction of miR-320a-3p levels in H295R cells significantly increased both mRNAs: CYP11A1 $(1.40 \pm 0.03$-fold; $p<0.01)$ and CYP17A1 $(1.53 \pm$ 0.09 -fold; $p<0.05)$.

3.5. miRNA Expression Differs in Normal Adrenal Tissue and Aldosterone-Producing Adenoma Tissue. In a previous study, 
StAR mRNA

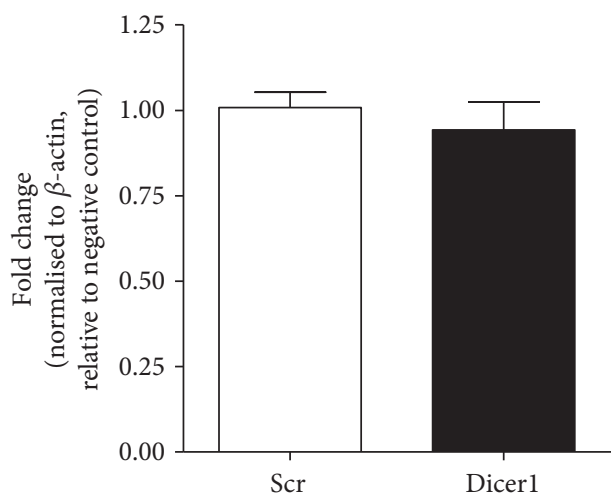

(a)

$3 \beta H S D I I$ mRNA

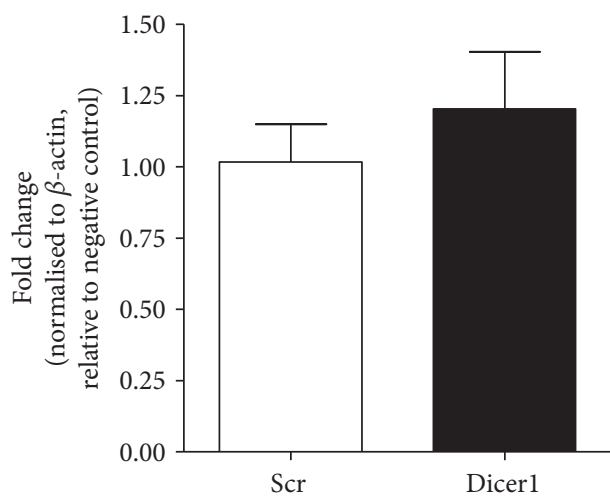

(c)

CYP17A1 mRNA

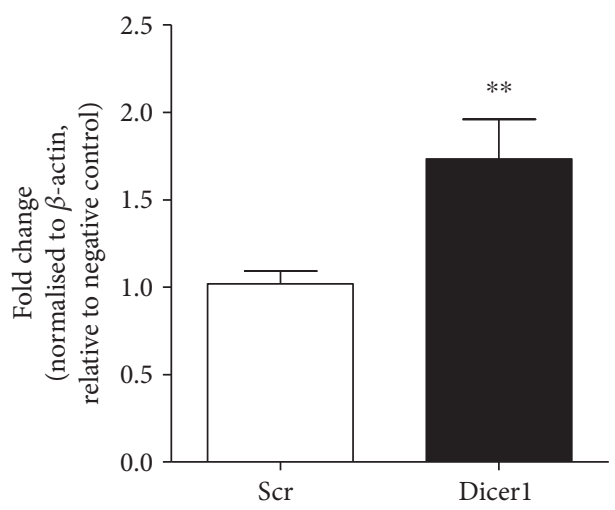

(e)
CYP11A1 mRNA

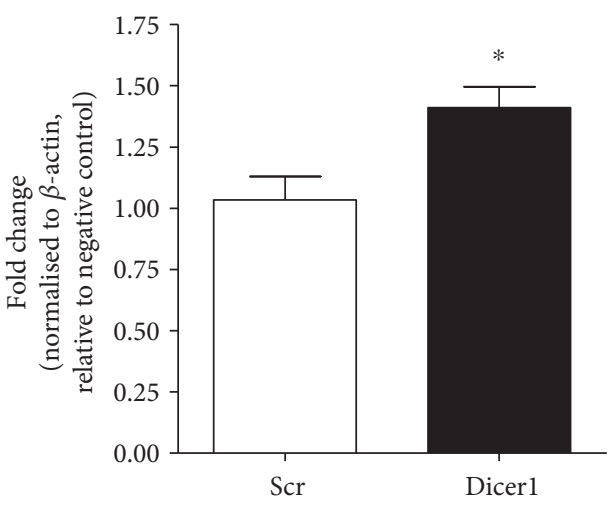

(b)

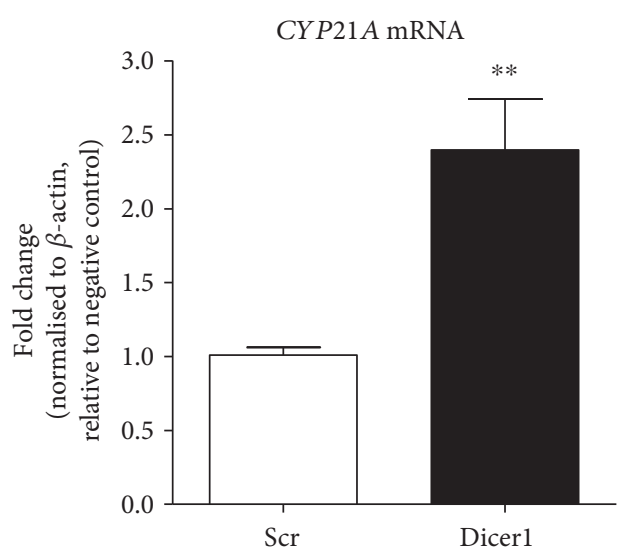

(d)

HSD $11 B 2$ mRNA

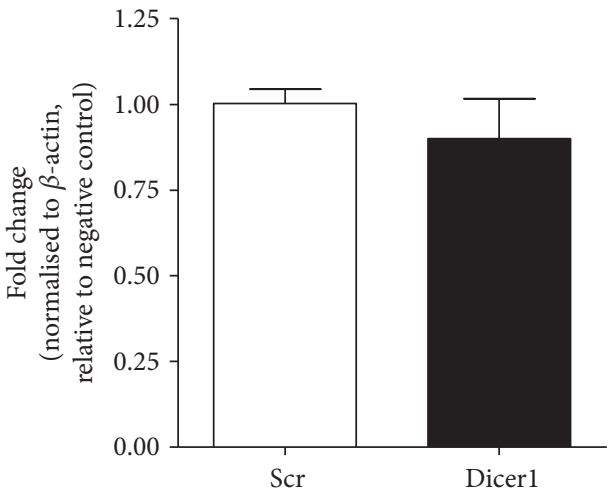

(f)

FIGURE 2: Results from Dicer1 siRNA transfection of H295R adrenocortical cells. Cells were transfected with Dicer1 siRNA or with a scrambled negative-control siRNA (mRNA levels for StAR (a), CYP11A1 (b), 3ßHSD11 (c), CYP21A (d), CYP17A1 (e), and HSD11B2 (f) were analysed 48 hours posttransfection by qRT-PCR). Cycle threshold values were normalised to $\beta$-actin mRNA and expressed relative to control cell levels. Results represent the mean of three independent biological experiments performed in triplicate \pm SEM; ${ }^{*} p<0.05$, ${ }^{* *} p<0.01$ compared to scrambled control.

miRNA expression was assessed by microarray analysis using total RNA samples isolated from four nondiseased human adrenal glands and four human aldosterone-producing adenomas (APAs) [8]. Of the five miRNAs investigated in the current study, two (miR-125a-5p and miR-495-3p) were expressed at significantly lower levels in APA tissue relative to nontumorous tissue; one (miR-320a-3p) was significantly increased in APA tissue and two (miR-125b-5p and miR-134-3p) did not differ significantly between the tissue types (Figure 6). 


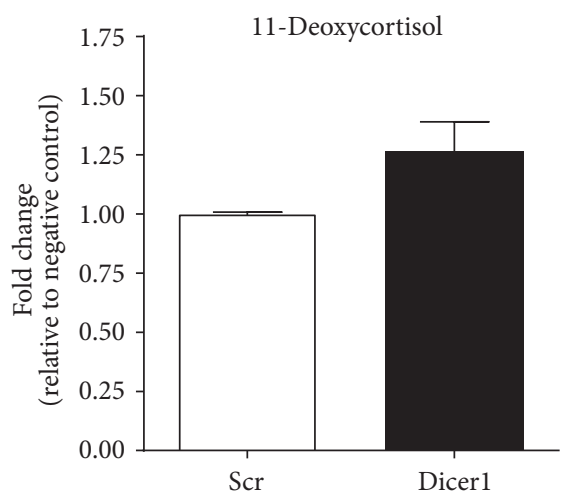

(a)

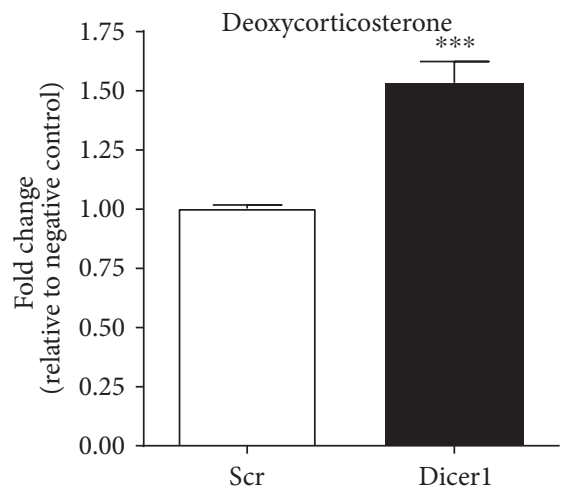

(d)

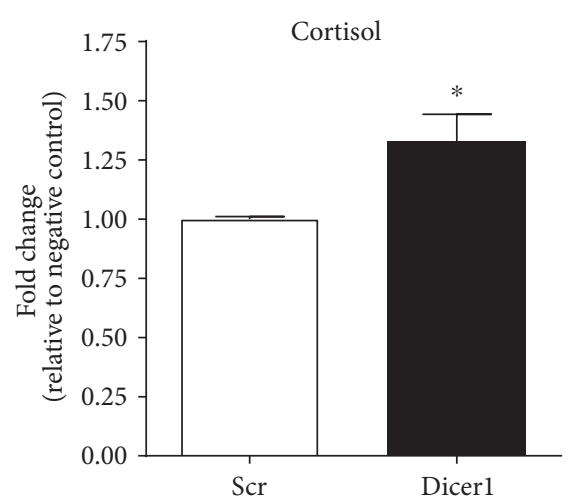

(b)

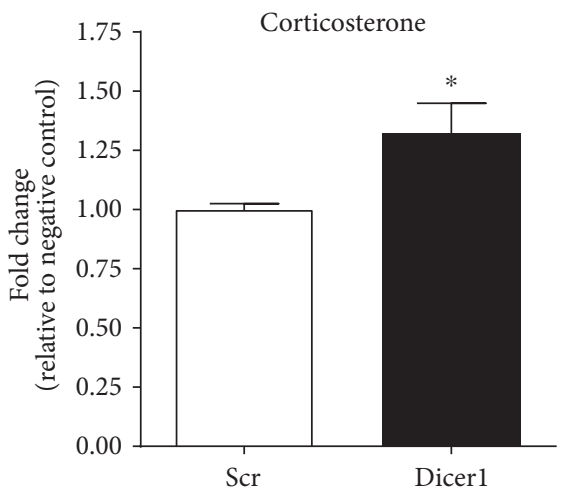

(e)

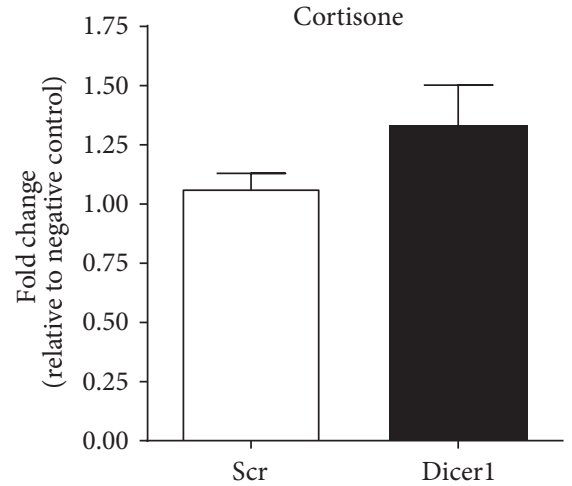

(c)

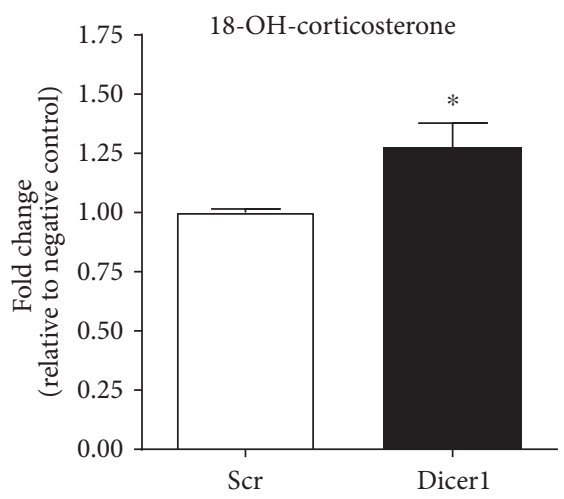

(f)

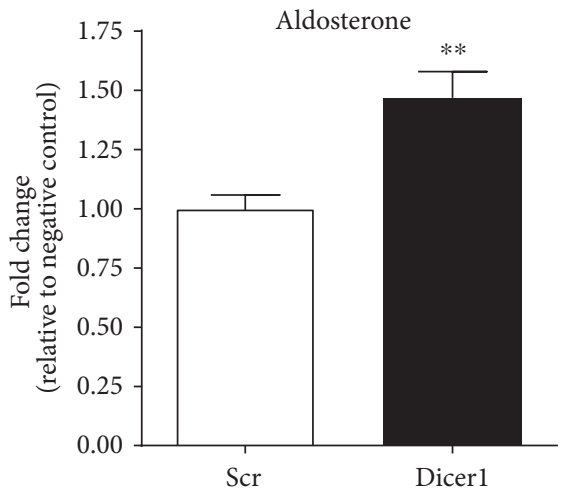

(g)

FIgure 3: Results from Dicer1 siRNA transfection of H295R adrenocortical cells. Cells were transfected with Dicer1 siRNA or with a scrambled negative-control siRNA. Cortisol-related steroid levels for 11-deoxycortisol (a), cortisol (b), and cortisone (c) and aldosteronerelated steroid levels for deoxycorticosterone (d), corticosterone (e), 18-OH-corticosterone (f), and aldosterone (g) were measured 48 hours posttransfection by LC MS:MS. Results represent the mean of three independent biological experiments performed in triplicate \pm SEM; ${ }^{*} p<0.05,{ }^{* *} p<0.01$, and ${ }^{* * *} p<0.001$ compared to scrambled control.

\section{Discussion}

The in vitro investigations conducted during this and our previous study [8] show that general reduction in miRNA levels through knockdown of Dicer1 significantly increases the abundance of all cytochrome P450-encoding mRNAs in this pathway (i.e., CYP11A1, CYP21A1, CYP17A1, CYP11B1, and $C Y P 11 B 2$ ). This confirms that miRNA can exert a net inhibitory effect on the expression of these key corticosteroidogenic genes which is reflected in altered steroid profile. Dicer1 knockdown increased levels of 11-deoxycortisol, corticosterone, 18-hydroxycorticosterone, cortisol, and aldosterone although the change in 11-deoxycortisol did not achieve statistical significance. The lack of effect on StAR expression has already been reported [27]. Interestingly, the dehydrogenase enzymes were not affected; the HSD11B1 and HSD11B2 products control the interconversion of cortisol and cortisone in many target tissues, but their mRNA levels were unaffected by Dicer 1 knockdown. Moreover, levels of cortisone were not significantly altered. These two genes and their respective products are not primary products of the adrenal cortex, and while these enzymes do not appear 
TABLE 2: Bioinformatic miRNA target site predictions for corticosteroidogenic genes.

\begin{tabular}{|c|c|c|c|c|c|c|c|c|c|c|}
\hline \multirow{2}{*}{$\begin{array}{l}\text { Gene } \\
\text { CYP11B1 }\end{array}$} & \multirow{2}{*}{$\begin{array}{c}\begin{array}{c}\text { 3'UTR length } \\
\text { (base pairs) }\end{array} \\
2022\end{array}$} & \multicolumn{2}{|c|}{ MicroRNA.org } & \multicolumn{2}{|c|}{ miRviewer } & \multicolumn{2}{|c|}{ TargetScan } & \multicolumn{2}{|c|}{$\begin{array}{l}\text { MicroCosm } \\
\text { targets }\end{array}$} & \multirow{2}{*}{$\begin{array}{c}\text { Unique miRNAs } \\
\text { with binding sites } \\
390\end{array}$} \\
\hline & & 151 & $(142)$ & 4 & (4) & 436 & (333) & 33 & (30) & \\
\hline CYP11B2 & 1428 & 89 & $(83)$ & 10 & $(10)$ & 323 & (253) & 6 & $(6)$ & 288 \\
\hline CYP11A1 & 213 & 26 & (26) & 1 & (1) & 51 & $(50)$ & 43 & (43) & 68 \\
\hline CYP17A1 & 171 & 24 & (24) & 0 & & 35 & $(34)$ & 23 & $(22)$ & 59 \\
\hline CYP21A2 & 508 & 57 & (56) & 11 & (10) & 78 & $(70)$ & 25 & (22) & 134 \\
\hline$H S D 3 B 2$ & 414 & 68 & (63) & 0 & & 73 & $(68)$ & 46 & $(44)$ & 125 \\
\hline StAR & 624 & 110 & $(101)$ & 4 & (4) & 273 & (244) & 11 & (11) & 285 \\
\hline HSD11B1 & 343 & 167 & (135) & 1 & (1) & 79 & $(72)$ & 70 & (65) & 159 \\
\hline HSD11B2 & 550 & 82 & (78) & 4 & (4) & 116 & (104) & 33 & (31) & 159 \\
\hline
\end{tabular}

The 3'UTR lengths of these 9 genes were identified using the UCSC Genome Browser Gateway and Ensembl Genome Browser. The number of predicted miRNA target sites from each database is listed, and the number of unique miRNAs predicted to bind each is shown in parentheses. The final column shows the cumulative number of miRNA target sites, with duplicates removed.

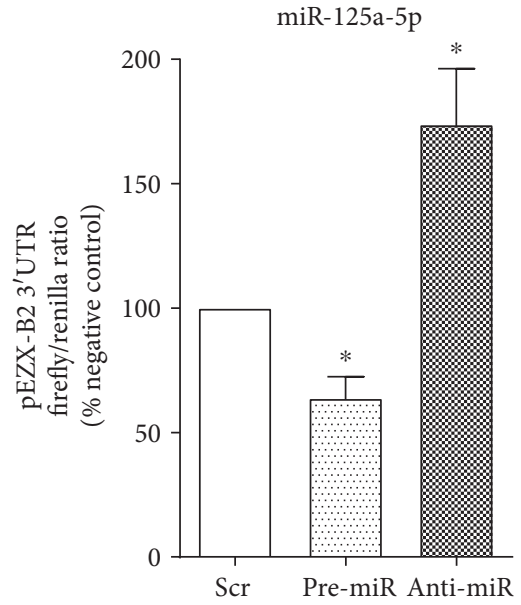

(a)

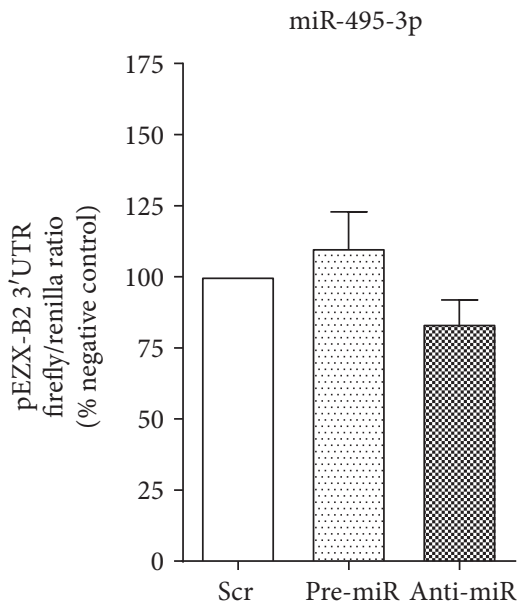

(d)

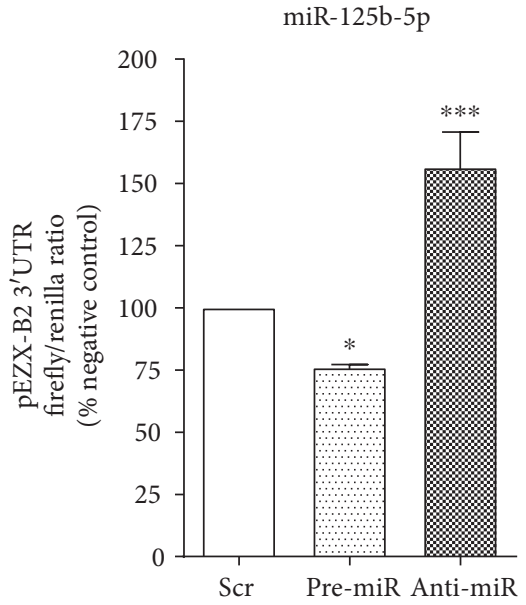

(b)

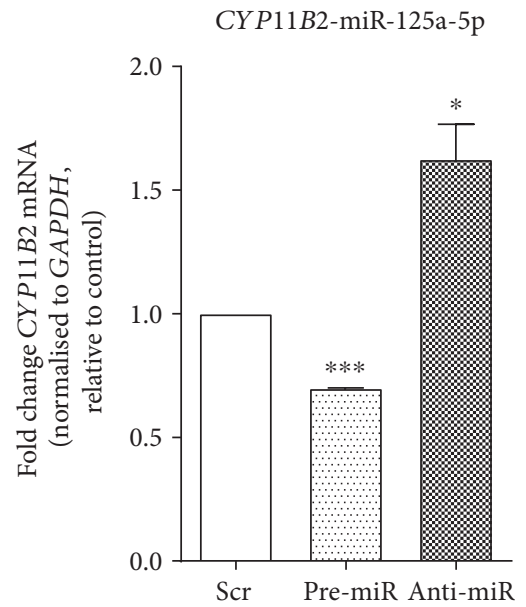

(e)

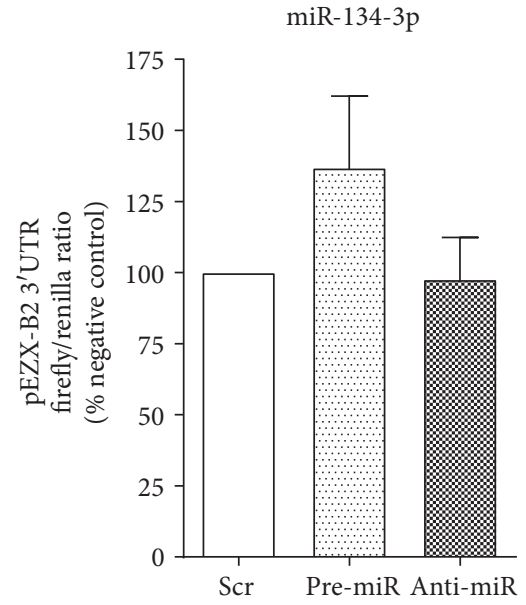

(c) CYP11B2-miR-125b-5p

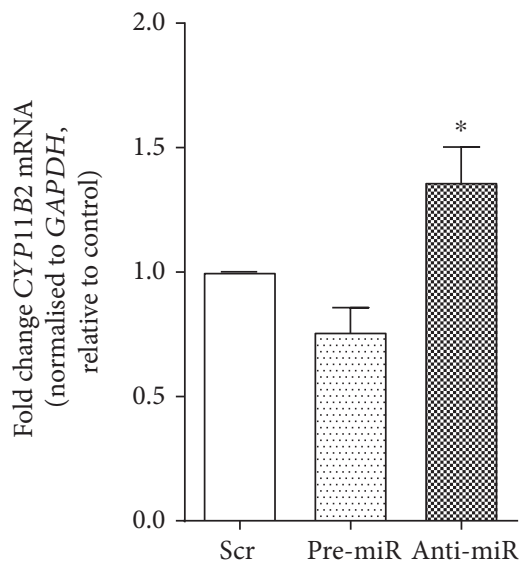

(f)

FIGURE 4: (a-d) Results from HeLa cells transfected with pEZX-B2 3'UTR reporter construct plasmids and either a specific miRNA Pre-miR or Anti-miR, or with a scrambled negative control RNA. Firefly and renilla luciferase luminescence was measured 48 hours posttransfection, and results are presented as firefly reporter gene luminescence normalised for renilla luminescence. H295R cells transfected with either miR-125a-5p (e) or miR-125b-5p (f) Pre-miR, miR-24 Anti-miR, or with a scrambled negative control RNA. CYP11B2 mRNA was measured 48 hours posttransfection by qRT-PCR, with cycle threshold values normalised to GAPDH mRNA and expressed relative to negative control. Results represent the mean of three independent biological experiments performed in triplicate $\pm S E M$; ${ }^{*} p<0.05$, ${ }^{* * *} p<0.001$ compared to negative control. 


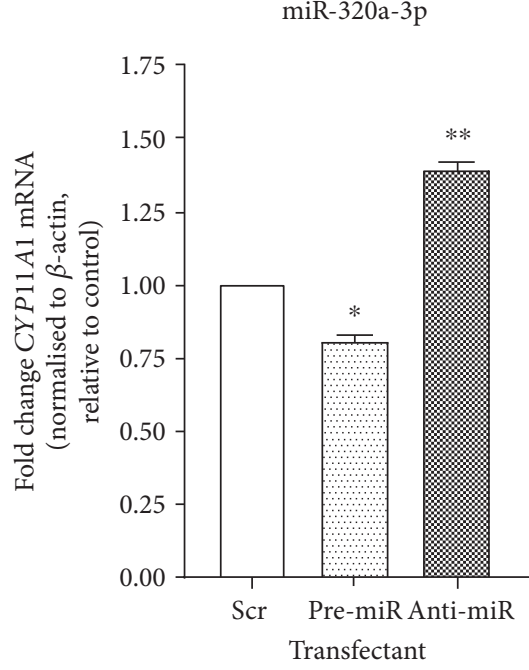

(a)
miR-320a-3p

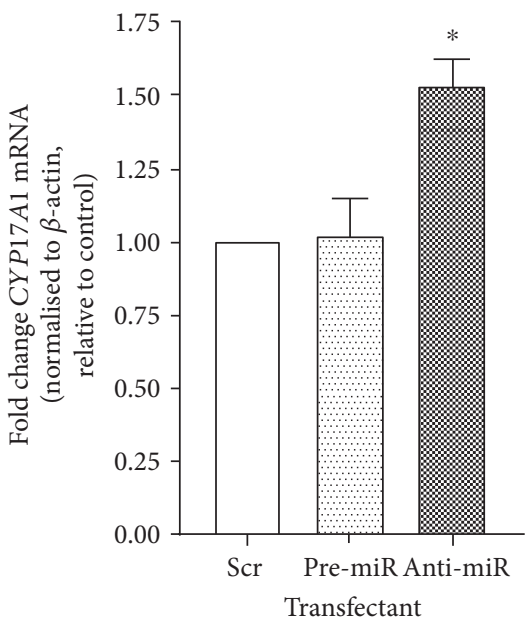

(b)

Figure 5: H295R cells transfected with miR-320a-3p Pre-miR, miR-320a-3p Anti-miR, or with a scrambled negative control RNA. CYP11A1 (a) or CYP17A1 (b) mRNA was measured 48 hours posttransfection by qRT-PCR, with cycle threshold values normalised to $\beta$-actin mRNA and expressed relative to negative control. Results represent the mean of three independent biological experiments performed in triplicate \pm SEM; ${ }^{*} p<0.05$ and ${ }^{* *} p<0.01$ compared to negative control.

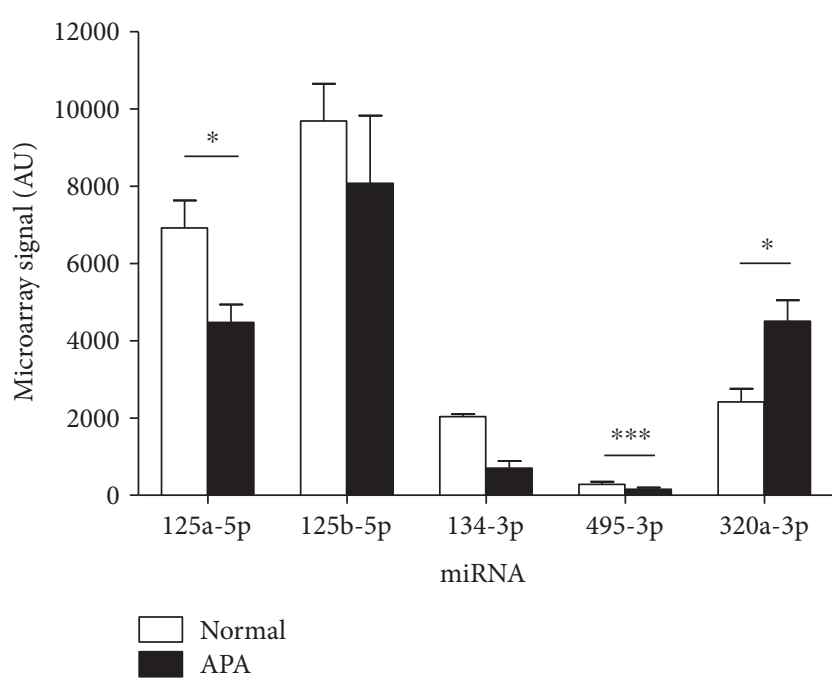

Figure 6: Expression levels of five miRNAs in normal adrenal tissue and APA tissue, as determined by microarray. Data show the normalised microarray signals, with a threshold value of $>500$ arbitrary units (AU) for at least one tissue type; ${ }^{*} p<0.05$, ${ }^{* * *} p<0.001$.

subject to miRNA regulation in H295R cells, other studies suggest miRNAs may regulate $H S D 11 B 1$ and $H S D 11 B 2$ in other tissues where they have greater functionality [28, 29]. These results indicate that a general inhibition of miRNA synthesis in H295R cells allows increased expression of most, but not all, corticosteroidogenic mRNA transcripts and a rise in steroid products.

Bioinformatic analysis identified putative miRNA target sites in all of the genes tested, although the number of sites predicted at each locus varied markedly depending on the algorithm used. This variability is well known [30], and so it is common to employ multiple algorithms in order to reduce error, as was the case here. These algorithms examine only 3'UTR sequences, and so do not identify possible miRNA binding sites within coding regions or 5'UTR $[31,32]$. Therefore, our studies were restricted to miRNA targeting of corticosteroidogenic mRNAs at the 3 'UTR. We also used bioinformatic tools to investigate whether the introns of these nine genes harbour any of the 1881 currently mapped human pri-miRNA sequences that occur throughout the genome, but none was found (data not shown). Therefore, any miRNA identified as controlling these genes is not cotranscribed with them.

Since bioinformatic analysis identified miRNA 3'UTR binding sites in all of the genes studied, it is not clear why some genes are apparently refractory, other than to note that predictive algorithms are not perfect, hence the need for experimental verification. Alternatively, structural differences may determine susceptibility; in this study, cytochrome P450 enzymes responded but dehydrogenases did not. Moreover, the 3'UTRs of the enzymes varied widely in their length and number of binding sites they were predicted to contain. Shorter 3'UTRs are thought to be more resistant to miRNA regulation [33].

miR-125a-5p and miR-125b-5p (but not miR-134-3p or miR-495-3p) altered CYP11B2 expression in vitro through direct targeting of its transcript 3'UTR. These two miRNAs belong to the same family, differing by only one nucleotide. However, miR-125a is located on human chromosome 19 in a cluster with miR-99b and let-7c, while miR-125b can originate from either human chromosome 11 (miR-125b-1) or human chromosome 21 (miR-125b-2). They are located intergenically, and miR-125b-2 is close to a gene encoding a long, noncoding RNA (lncRNA). The miR-125 family has been implicated in the regulation of several cell types and has many confirmed targets, including vascular cell cycle- 
related genes of importance in hypertension [34, 35]. CYP11A1 and CYP17A1 are both targets for miR-320a-3p. Overexpression of miR-320-3p did not significantly affect CYP17A1 mRNA levels but inhibition enhanced levels of both mRNAs; this implies that CYP17A1 is already under maximal inhibition at the miR-320-3p target site. CYP17A1 determines relative flow of steroid biosynthesis through the 17-hydroxy and 17-deoxy pathways. An influence here might affect mineralocorticoid-glucocorticoid balance. Like most miRNAs characterised to date, miR-320-3p has been reported to have multiple mRNA targets and regulate different pathways in different cell types. For example, it has been implicated as a tumor suppressor in leukaemia [36] but has also been associated with a detrimental effect in cerebral ischaemia [37].

These results show the potential for several miRNAs to modulate corticosteroidogenesis at the majority of relevant loci. It is not possible to ascertain from these data whether the overall effect is due to several small individual effects or whether action at one locus predominates. We believe there are many more miRNA-mediated effects on this pathway than have been identified thus far, and it may be that the individual miRNA effects we have investigated here are not the most significant. This is borne out by the fact that qRT-PCR analysis of specific miRNA levels in the Dicer1 knockdown cells showed no significant reduction on the levels of the miRNAs miR-125a-5p, miR-125b-5p, miR134-3p, miR-320a-3p, and miR-495-3p relative to controls (data not shown). While the Dicer1 knockdown experiments were not designed with the intention of analysing individual miRNA levels, or powered accordingly, it is tempting to speculate that other miRNAs undergoing a greater dynamic shift as a result of Dicer1 knockdown will emerge as those most responsible for the observed changes in phenotype. Importantly, if miRNAs are to be incorporated into the existing model of control, more information on the control of their own production is required. For example, it was previously shown that miR-21 levels increase in H295R cells following angiotensin II stimulation [10]. It must also be emphasised that the H295R cell line is derived from neoplastic tissue with an atypical steroid profile [24]. Also, in dispersed cell experiments, the strict zonal constraints on function and gene expression of the intact gland (e.g., the zonally distinct expressions of CYP11B1 and CYP11B2) are absent. Nevertheless, the H295R cell has proved a valuable guide to in vivo adrenal physiology [24].

There is obvious interest in assessing the significance of such miRNA repression in vivo. We compared the expression profiles of miRNAs in normal adrenal and APA tissue and identified differential expression of five miRNAs, in agreement with previous studies [8, 17-23]. miR-125a-5p expression was lower in APA. APA secretes high levels of aldosterone, and the study described above showed CYP11B2 to be affected by this miRNA. However, its clustered miRNAs were each more abundant in APA tissue than in normal adrenal tissue. Previous studies report reduced CYP17A1 expression in APA [38]. Our study has shown a high level of miR-320a-3p in the carcinoma-derived $\mathrm{H} 295 \mathrm{R}$ cell line and its potency as a
CYP17A1 regulator. miRNA targeting of transcription may be a common feature of this condition.

In summary, by manipulating their levels, we have shown that miRNAs affect the expression of multiple corticosteroidogenic genes and thereby affect the steroid profile at multiple points. Corticosteroidogenic gene sequences include $3^{\prime} U T R$ sites that enable their posttranscriptional regulation by specific miRNAs, as demonstrated here for CYP11B2, CYP11A1, and CYP17A1. Importantly, a miRNA which affects aldosterone synthesis and another that affects CYP17A1 are expressed at different levels in normal adrenal and APA tissue suggesting relevance to this form of adrenal pathology.

\section{Data Access}

All data are included in the manuscript, and requests for additional information should be made to the corresponding author.

\section{Conflicts of Interest}

The authors have nothing to disclose.

\section{Acknowledgments}

The authors are grateful to Professor William E. Rainey (Medical College of Georgia, Augusta, GA) for the kind gift of H295R cells, to Paul Stewart (University of Leeds) for providing the normal human adrenal tissue, and to Tim Harvey (University of Glasgow) for his technical assistance in the tissue preparation. This work was funded by the British Heart Foundation Project Grant PG/09/092, awarded to Eleanor Davies and Scott M. MacKenzie. Louise A. Diver was supported by the College of Medical, Veterinary and Life Sciences Medical Research Council Doctoral Training Grant Scholarship (Grant no. G0900185-1/1). Ayesha Ejaz was supported by the Society for Endocrinology Summer Studentship. Eleanor Davies is a member of COST Action BM1301 Aldosterone and Mineralocorticoid Receptor (ADMIRE). Eleanor Davies and Scott M. MacKenzie are participants in the EU-H2020 funded project ENSAT-HT.

\section{References}

[1] G. Arnaldi, A. Angeli, A. B. Atkinson et al., "Diagnosis and complications of Cushing's syndrome: a consensus statement," The Journal of Clinical Endocrinology and Metabolism, vol. 88, no. 12, pp. 5593-5602, 2003.

[2] P. Milliez, X. Girerd, P. F. Plouin, J. Blacher, M. E. Safar, and J. J. Mourad, "Evidence for an increased rate of cardiovascular events in patients with primary aldosteronism," Journal of the American College of Cardiology, vol. 45, no. 8, pp. 1243-1248, 2005.

[3] D. G. Levy, R. Rocha, and J. W. Funder, "Distinguishing the antihypertensive and electrolyte effects of eplerenone," The Journal of Clinical Endocrinology and Metabolism, vol. 89, no. 6, pp. 2736-2740, 2004.

[4] J. M. Connell and E. Davies, "The new biology of aldosterone," The Journal of Endocrinology, vol. 186, no. 1, pp. 1-20, 2005. 
[5] J. W. Funder, P. T. Pearce, R. Smith, and A. I. Smith, "Mineralocorticoid action: target tissue specificity is enzyme, not receptor, mediated," Science, vol. 242, no. 4878, pp. 583$585,1988$.

[6] C. R. Edwards, P. M. Stewart, D. Burt et al., "Localisation of 11 beta-hydroxysteroid dehydrogenase-tissue specific protector of the mineralocorticoid receptor," Lancet, vol. 2, no. 8618, pp. 986-989, 1988.

[7] M. Young, M. Fullerton, R. Dilley, and J. Funder, "Mineralocorticoids, hypertension, and cardiac fibrosis," The Journal of Clinical Investigation, vol. 93, no. 6, pp. 2578-2583, 1994.

[8] S. Robertson, S. M. MacKenzie, S. Alvarez-Madrazo et al., "MicroRNA-24 is a novel regulator of aldosterone and cortisol production in the human adrenal cortex," Hypertension, vol. 62 , no. 3, pp. 572-578, 2013.

[9] C. C. Huang and H. H. Yao, "Inactivation of Dicer1 in steroidogenic factor 1-positive cells reveals tissue-specific requirement for Dicer1 in adrenal, testis, and ovary," BMC Developmental Biology, vol. 10, p. 66, 2010.

[10] D. G. Romero, M. W. Plonczynski, C. A. Carvajal, E. P. Gomez-Sanchez, and C. E. Gomez-Sanchez, "Microribonucleic acid-21 increases aldosterone secretion and proliferation in H295R human adrenocortical cells," Endocrinology, vol. 149, no. 5, pp. 2477-2483, 2008.

[11] S. Nusrin, S. K. Tong, G. Chaturvedi, R. S. Wu, J. P. Giesy, and R. Y. Kong, "Regulation of CYP11B1 and CYP11B2 steroidogenic genes by hypoxia-inducible miR-10b in H295R cells," Marine Pollution Bulletin, vol. 85, no. 2, pp. 344-351, 2014.

[12] B. C. Schanen and X. Li, "Transcriptional regulation of mammalian miRNA genes," Genomics, vol. 97, no. 1, pp. 1-6, 2011.

[13] M. Ha and V. N. Kim, "Regulation of microRNA biogenesis," Nature Reviews. Molecular Cell Biology, vol. 15, no. 8, pp. 509-524, 2014.

[14] T. Thum and M. Mayr, "Review focus on the role of microRNA in cardiovascular biology and disease," Cardiovascular Research, vol. 93, no. 4, pp. 543-544, 2012.

[15] J. R. Kemp, H. Unal, R. Desnoyer, H. Yue, A. Bhatnagar, and S. S. Karnik, "Angiotensin II-regulated microRNA 483-3p directly targets multiple components of the renin-angiotensin system," Journal of Molecular and Cellular Cardiology, vol. 75, pp. 25-39, 2014.

[16] M. D. Jansson and A. H. Lund, "MicroRNA and cancer," Molecular Oncology, vol. 6, no. 6, pp. 590-610, 2012.

[17] P. S. Soon, L. J. Tacon, A. J. Gill et al., "miR-195 and miR-483$5 p$ identified as predictors of poor prognosis in adrenocortical cancer," Clinical Cancer Research, vol. 15, no. 24, pp. 76847692, 2009.

[18] E. I. Bimpaki, D. Iliopoulos, A. Moraitis, and C. A. Stratakis, "MicroRNA signature in massive macronodular adrenocortical disease and implications for adrenocortical tumourigenesis," Clinical Endocrinology, vol. 72, no. 6, pp. 744-751, 2010.

[19] D. Iliopoulos, E. I. Bimpaki, M. Nesterova, and C. A. Stratakis, "MicroRNA signature of primary pigmented nodular adrenocortical disease: clinical correlations and regulation of Wnt signaling," Cancer Research, vol. 69, no. 8, pp. 32783282, 2009.

[20] Z. Tombol, P. M. Szabó, V. Molnár et al., "Integrative molecular bioinformatics study of human adrenocortical tumors: microRNA, tissue-specific target prediction, and pathway analysis," Endocrine-Related Cancer, vol. 16, no. 3, pp. 895906, 2009.
[21] K. J. Schmitz, J. Helwig, S. Bertram et al., "Differential expression of microRNA-675, microRNA-139-3p and microRNA335 in benign and malignant adrenocortical tumours," Journal of Clinical Pathology, vol. 64, no. 6, pp. 529-535, 2011.

[22] D. Velazquez-Fernandez, S. Caramuta, D. M. Özata et al., "MicroRNA expression patterns associated with hyperfunctioning and non-hyperfunctioning phenotypes in adrenocortical adenomas," European Journal of Endocrinology, vol. 170, no. 4, pp. 583-591, 2014.

[23] L. Lenzini, B. Caroccia, A. G. Campos et al., "Lower expression of the TWIK-related acid-sensitive K+channel 2 (TASK-2) gene is a hallmark of aldosterone-producing adenoma causing human primary aldosteronism," The Journal of Clinical Endocrinology and Metabolism, vol. 99, no. 4, pp. E674-E682, 2014.

[24] W. E. Rainey, I. M. Bird, and J. I. Mason, "The NCI-H295 cell line: a pluripotent model for human adrenocortical studies," Molecular and Cellular Endocrinology, vol. 100, no. 1-2, pp. 45-50, 1994.

[25] M. C. Ingram and R. Fraser, "Plasma corticosteroid profiling: brief opinion of its current status in clinical diagnosis and research," Expert Review of Endocrinology and Metabolism, vol. 5, no. 2, pp. 181-188, 2010.

[26] K. J. Livak and T. D. Schmittgen, "Analysis of relative gene expression data using real-time quantitative PCR and the 2(-delta delta $\mathrm{C}(\mathrm{T})$ ) method," Methods, vol. 25, no. 4, pp. 402-408, 2001.

[27] Z. Hu, W. J. Shen, Y. Cortez et al., "Hormonal regulation of microRNA expression in steroid producing cells of the ovary, testis and adrenal gland," PloS One, vol. 8, no. 10, article e78040, 2013.

[28] Y. Han, C. A. Staab-Weijnitz, G. Xiong, and E. Maser, "Identification of microRNAs as a potential novel regulatory mechanism in HSD11B1 expression," The Journal of Steroid Biochemistry and Molecular Biology, vol. 133, pp. 129-139, 2013.

[29] M. Rezaei, T. Andrieu, S. Neuenschwander et al., "Regulation of 11beta-hydroxysteroid dehydrogenase type 2 by microRNA," Hypertension, vol. 64, no. 4, pp. 860-866, 2014.

[30] T. M. Witkos, E. Koscianska, and W. J. Krzyzosiak, "Practical aspects of microRNA target prediction," Current Molecular Medicine, vol. 11, no. 2, pp. 93-109, 2011.

[31] Y. Tay, J. Zhang, A. M. Thomson, B. Lim, and I. Rigoutsos, "MicroRNAs to Nanog, Oct4 and Sox 2 coding regions modulate embryonic stem cell differentiation," Nature, vol. 455, no. 7216, pp. 1124-1128, 2008.

[32] J. R. Lytle, T. A. Yario, and J. A. Steitz, “Target mRNAs are repressed as efficiently by microRNA-binding sites in the $5^{\prime}$ UTR as in the $3^{\prime}$ UTR," Proceedings of the National Academy of Sciences of the United States of America, vol. 104, no. 23, pp. 9667-9672, 2007.

[33] A. Stark, J. Brennecke, N. Bushati, R. B. Russell, and S. M. Cohen, "Animal microRNAs confer robustness to gene expression and have a significant impact on 3'UTR evolution," Cell, vol. 123, no. 6, pp. 1133-1146, 2005.

[34] Y. M. Sun, K. Y. Lin, and Y. Q. Chen, "Diverse functions of miR-125 family in different cell contexts," Journal of Hematology \& Oncology, vol. 6, p. 6, 2013.

[35] D. Li, P. Yang, Q. Xiong et al., "MicroRNA-125a/b-5p inhibits endothelin-1 expression in vascular endothelial cells," Journal of Hypertension, vol. 28, no. 8, pp. 1646-1654, 2010.

[36] Z. Xishan, L. Ziying, D. Jing, and L. Gang, "MicroRNA-320a acts as a tumor suppressor by targeting BCR/ABL oncogene 
in chronic myeloid leukemia," Scientific Reports, vol. 5, article 12460, 2015.

[37] S. Sepramaniam, A. Armugam, K. Y. Lim et al., "MicroRNA 320a functions as a novel endogenous modulator of aquaporins 1 and 4 as well as a potential therapeutic target in cerebral ischemia," The Journal of Biological Chemistry, vol. 285, no. 38, pp. 29223-29230, 2010.

[38] T. Wang, F. Satoh, R. Morimoto et al., "Gene expression profiles in aldosterone-producing adenomas and adjacent adrenal glands," European Journal of Endocrinology, vol. 164, no. 4, pp. 613-619, 2011. 


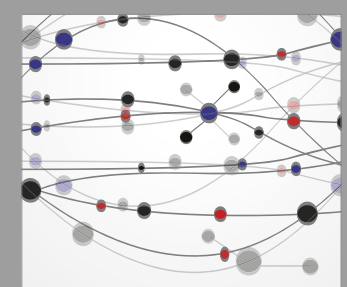

The Scientific World Journal
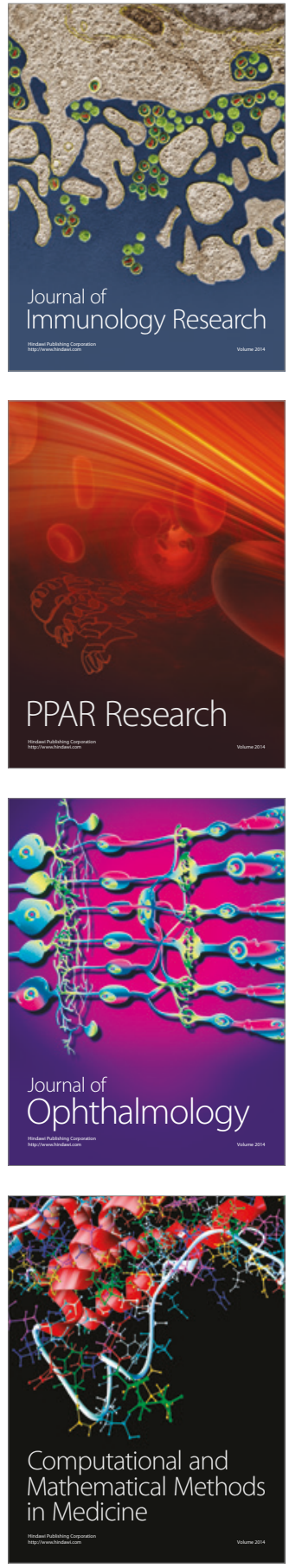

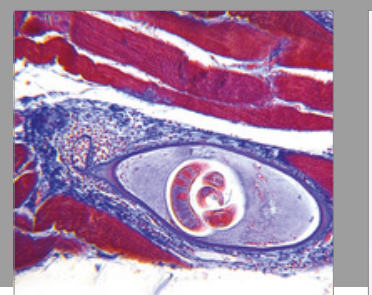

Gastroenterology Research and Practice
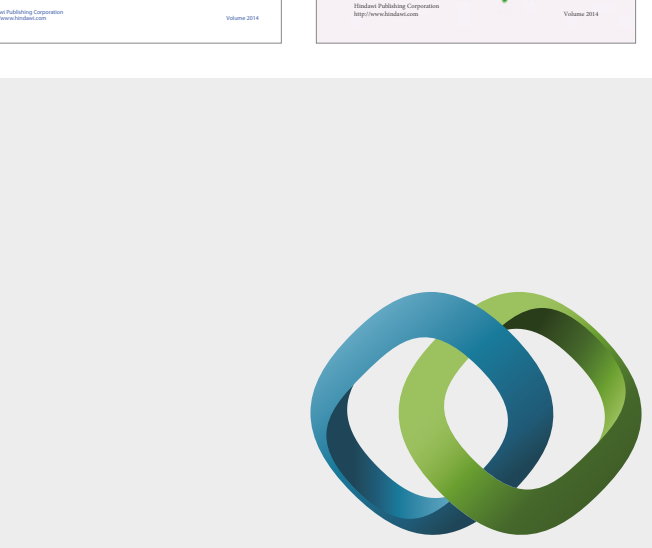

\section{Hindawi}

Submit your manuscripts at

https://www.hindawi.com
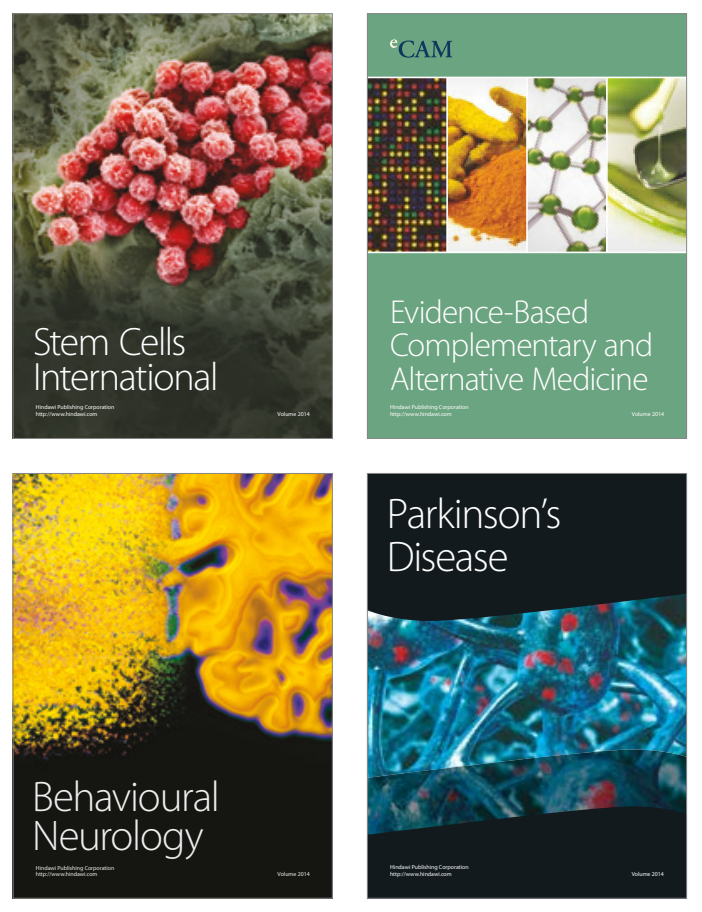
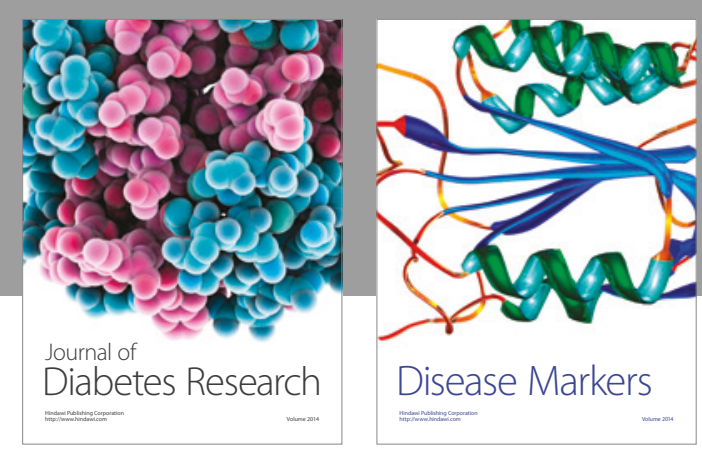

Disease Markers
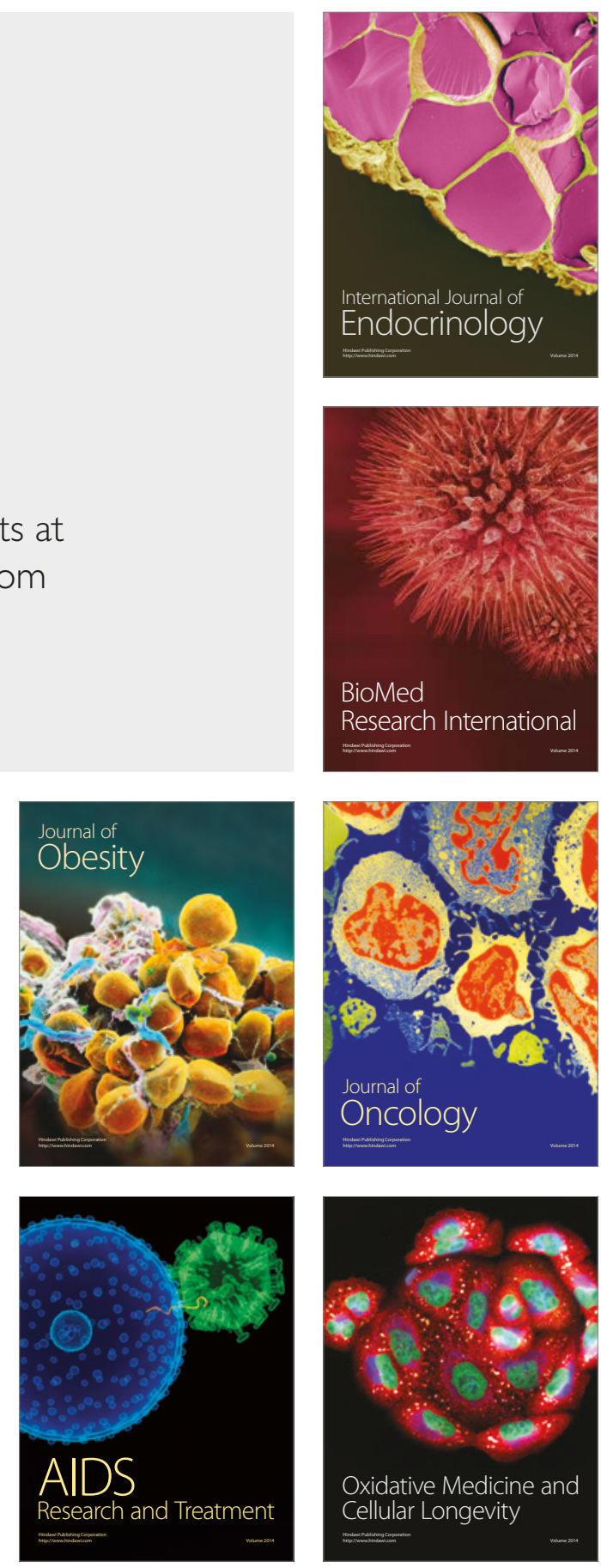